
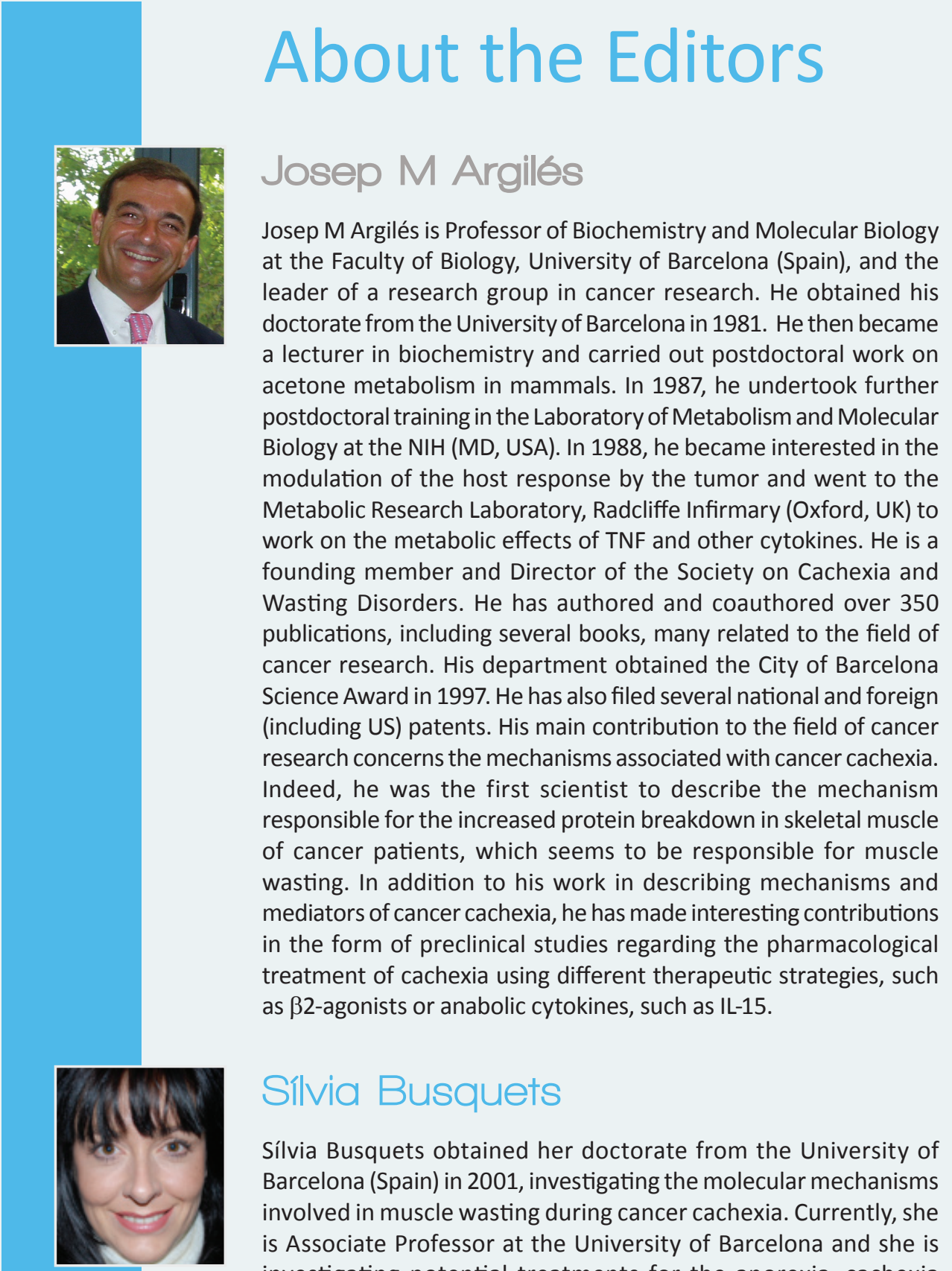

\title{
Sílvia Busquets
}

Sílvia Busquets obtained her doctorate from the University of Barcelona (Spain) in 2001, investigating the molecular mechanisms involved in muscle wasting during cancer cachexia. Currently, she is Associate Professor at the University of Barcelona and she is investigating potential treatments for the anorexia-cachexia syndrome. 


\section{Foreword}

\section{Cancer cachexia}

\section{Josep M Argilés \& Sílvia Busquets}

Cancer cachexia is a multiorganic syndrome associated with neoplastic syndromes, characterized by a marked weight loss, anorexia, asthenia and anemia. Cachexia is invariably associated with the presence and growth of the tumor and leads to a malnutrition status due to the induction of anorexia or decreased food intake. It occurs in the majority of cancer patients before death and it is responsible for the deaths of a large number of cancer patients.

The competition for nutrients between the tumor and the host leads to an accelerated catabolism state, which promotes severe metabolic disturbances in the host, including hypermetabolism, which leads to an increased energetic inefficiency. The abnormalities associated with cancer cachexia include anorexia, weight loss, muscle loss and atrophy, anemia and alterations in carbohydrate, lipid and protein metabolism. The degree of cachexia is inversely correlated with the survival time of the patient and it always implies a poor prognosis.

Fatigue, as a result of muscle wasting, is an extremely common symptom in patients with cancer cachexia; this condition is observed among a high percentage of advanced cancer patients. Inflammatory cytokines are involved in the wasting process associated with cachexia. These include IL6 , TNF- $\alpha$, IL-1 $\beta$ and IFN- $\gamma$. Although many inflammatory mediators may play a role in muscle wasting during cachexia, these cytokines have emerged as a critical factor related to the maintenance of body mass during disease. Although there may be many different tissues and cell types that are 
responsible for the increase in circulating cytokines during some types of cancer, there is evidence that the tumors are an important source. Cytokines by themselves are capable of inducing weight loss. Although a plethora of treatments for the cachectic syndrome have been proposed, unfortunately not a single one is completely satisfactory.

When treating weight loss associated with cachexia, two targets have to be considered. First, food intake should be controlled with the aim of reducing anorexia. In addition, contributing factors to decreased food intake could be assessed and treated. This clearly constitutes the first aim of the therapy of wasting. Second, providing complete nutritional requirements by means of total parenteral nutrition does not abrogate the weight loss. Thus, it is clear that in addition to controlling food intake, the metabolic disturbances associated with tumor burden contribute most importantly to the appearance of cachexia. Therefore, neutralizing the metabolic alterations, which include abnormal carbohydrate metabolism, lipid mobilization, hepatic protein metabolism and, above all, alterations in the rate of skeletal muscle protein breakdown, is of paramount importance. The best treatment of the cachectic syndrome is most likely to be a multifactorial approach. In relation to this, the combination of nutritional support with different nutraceuticals has already been reported to be relatively successful. A combination of nutritional support and nutraceuticals with specific drugs may lead to optimal results. There are two objectives of any therapeutic combination: an anticatabolic aim directed towards both fat and muscle catabolism and an anabolic objective leading to the synthesis of macromolecules, such as contractile proteins.

The five chapters in this book review and summarize the current status of cancer cachexia and its therapy. The authors have addressed a variety of relevant clinical issues, with the initial chapter by Johns and coworkers reviewing prevalence and clinical features of the cachectic syndrome in cancer patients. Following suit, the second chapter deals with the pathogenesis of the syndrome, taking into account all of the factors involved, both related to food intake and metabolic disturbances. Chapter 3 elegantly reviews the present therapeutic approaches available with special emphasis on pharmacological combinations. Chapter 4 analyzes the drugs currently involved in clinical trials that may have a future potential in the treatment of the wasting syndrome in cancer. Finally, Chapter 5 deals with comorbidities and prognosis. In summary, the authors have provided a comprehensive review of the mechanisms, mediators and treatment of cancer cachexia. However, more research into both the clinics of the syndrome and the mechanisms underlying the activity of the novel pharmacological agents is still needed. 
Financial \& competing interests disclosure

JM Argilés is a Consultant for Danone, Rottapharm and Novartis. He has also received honoraria from Danone, Rottapharm, Abbott, Novartis, Amgen, Merck Sharp \& Dohme and Par Pharmaceuticals. The authors have no other relevant affiliations or financial involvement with any organization or entity with a financial interest in or financial conflict with the subject matter or materials discussed in the manuscript apart from those disclosed.

No writing assistance was utilized in the production of this manuscript. 